\title{
Development of a Novel Embedded Control System for Decision Making During Collision Avoidance Conditions
}

\author{
M.Hariharan ${ }^{1}$, S.Priya ${ }^{2}$ \\ 1,2 Department of Electrical and Electronics Engineering, \\ Anand Institute of Higher Technology Kazhipattur, Chennai- 603103, Tamilnadu, India
}

\begin{abstract}
Road traffic accidents are one of the world's largest public health problems. To reduce these numbers, vehicle manufacturers are developing systems that can detect hazardous traffic situations and actively assist road users in avoiding or mitigating accidents. Collision Avoidance (CA) Mechanism is being identified as a suitable method for mitigating the road accidents. The main objective is to make the driver to attend only the emergency calls. Initially when a call is made to driver, he will receive a message intimating that the person is driving. If once again he calls the driver then it is understood that it is emergency. So the driver will get an intimation regarding the emergency call in voice and automatically left indicator of the car will be turned on. This system will prevent accidents due to phone calls. In addition to this facility, the proposed system also helps to detect the obstacles behind the vehicle while reversing and alarms the driver if the both hands are out of the grip from the steering while driving.
\end{abstract}

Keywords: Collision Avoidance (CA), Radio Frequency (RF), Universal Asynchronous Receiver Transmitter (UART), Special Function Register (SFR), Signal to Noise Ratio (SNR), Surface Acoustic Wave (SAW)

\section{INTRODUCTION}

Road traffic accidents are one of the world's largest public health problems. In the European Union alone, traffic accidents cause approximately 1.8 million injuries and 43,000 fatalities each year. To reduce these numbers, vehicle manufacturers are developing systems that can detect hazardous traffic situations and actively assist road users in avoiding or mitigating the road accidents. Systems that assist drivers in avoiding collisions are becoming increasingly more common and are even being introduced as standard equipment in some passenger cars. Collision avoidance (CA) systems can generally be divided into three layers, Measurements from onboard sensors, such as accelerometers, gyroscope, and radars, are processed in the first layer and then interpreted in the second layer, which makes decisions on when and how to assist the driver. The third layer executes the decision, e.g., by automatically applying the brakes of the vehicle.

The measurements contain random errors, and consequently, the vehicle and object state estimates that are obtained in the sensor fusion layer are associated with uncertainties. A threat assessment algorithm utilizes these estimates to make predictions of road-user trajectories. Based on these predictions, an assessment is performed to estimate if and how a potential accident can be prevented. Both the state estimates and the predictions are associated with uncertainties that need to be treated properly. Moreover, to obtain a high customer acceptance, it is important that the system also accounts for the preferences of driver, such that the driver is not disturbed by the system during normal driving conditions.

\subsection{EXISTING SYSTEM}

\section{LITERATURE SURVEY}

Goodrich and Boer proposed that CA systems should account for not only the capabilities of the vehicle and the sensor system but also the autonomy and preferences of the driver. Benefit and cost functions are introduced to make decisions based on a tradeoff between the potential benefit of an intervention and the cost of disturbing the driver with an unnecessary intervention. Driving is a serious responsibility with physical and mental abilities impacting on the driving activities of a driver. It is more than moving a vehicle and knowing how to use the accelerator, brake pedals and steering. Driving is at its best when you have knowledge and required skills to drive competently in accordance with those rules and regulations guiding the public roads. If we consider, previous technologies will not give hands for the perfect action, it should not be done in the mean time.

\section{DEMERITS OF THE EXISTING SYSTEM}

- No automatic speed control

- Less Sensors Fusion

- Cannot predict the CA 


\subsection{PROPOSED SYSTEM}

The objective of CA systems is to assist drivers in avoiding collisions with other road users and objects, without triggering interventions that the driver may consider unnecessary. Clearly, to autonomously avoid a collision, an intervention must be triggered while an accident is still avoidable. However, most collisions that can be avoided by CA systems possibly can also be avoided by a skilled driver. This implies that there is no way of predicting whether the driver would have been able to handle the situation without the system intervening.

\section{MERITS}

- Automatic control.

- Quick Recovery.

- Indication of the distance to avoid the collision.

- Hand free communication

- Enabling Serial Communication between the RS485 ports of AT89S52 Controller

\section{Block Diagram of TX/RX Section of the CA System} TRANSMITTER:

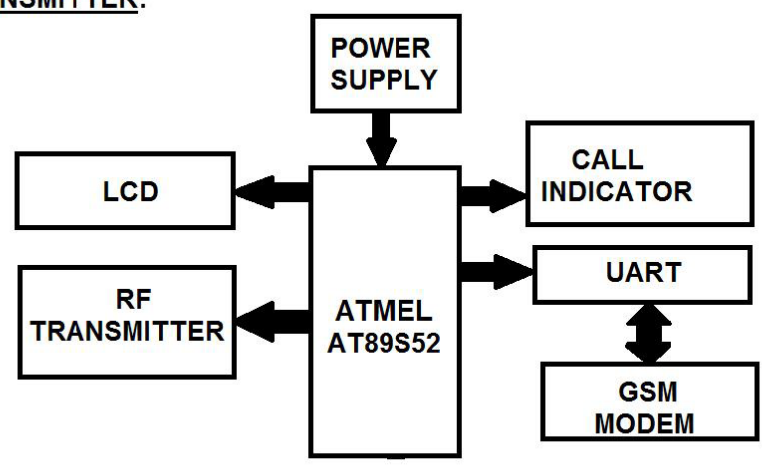

Fig 2.2.1: Block Diagram of Transmitter Section

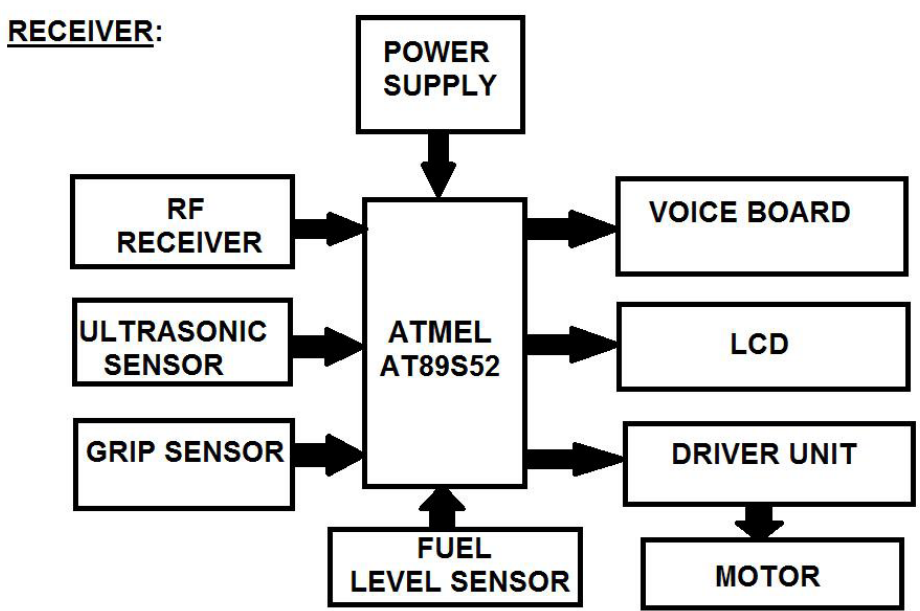

Fig 2.2.2: Block Diagram of Receiver Section

\section{HARDWARE DESIGN DETAILS}

\subsection{THE ATMEL AT89S52 MICROCONTROLLER}

The AT89S52 is a low-power, high-performance CMOS 8-bit microcontroller with $8 \mathrm{~K}$ bytes of insystem programmable Flash memory. It provides high cost- effective solutions to the design of complex embedded control systems. The device is manufactured using Atmel's high-density nonvolatile memory technology and is compatible with the Industry standard 80C51 instruction set and pin out configuration. 


\section{0-lead PDIP}

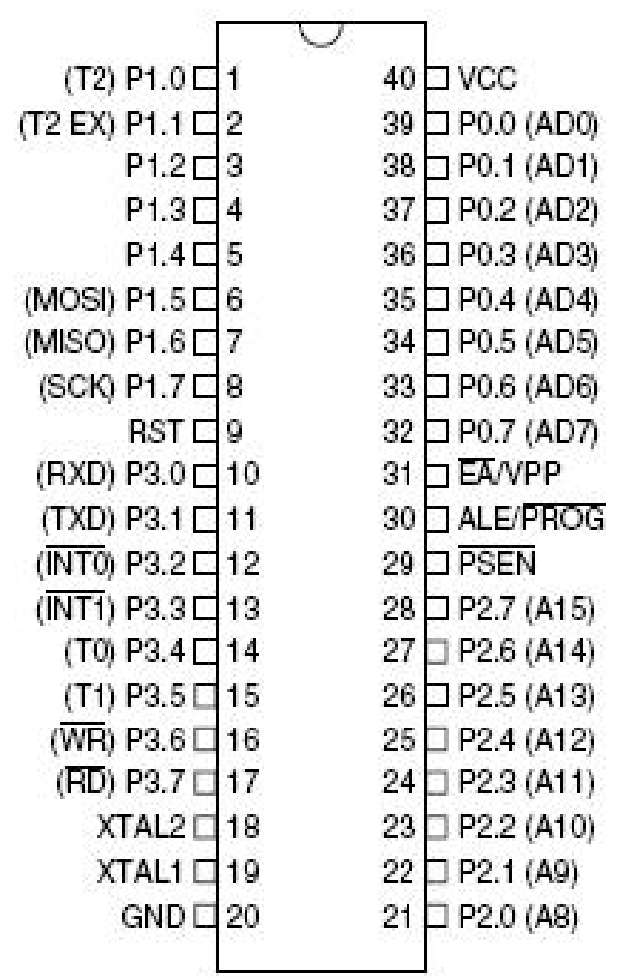

Fig 3.1.1 Functional Pin Diagram of AT89S52 Microcontroller

\subsubsection{FEATURES OF AT89S52}

- Compatible with MCS-51 Products.

- $\quad$ KK Bytes of In-System Programmable (ISP) Flash Memory - Endurance: 1000 Write/Erase Cycles.

- $\quad 4.0 \mathrm{~V}$ to $5.5 \mathrm{~V}$ Operating Range.

- Fully Static Operation: $0 \mathrm{~Hz}$ to $33 \mathrm{MHz}$

- Three-level Program Memory Lock.

- 256 x 8-bit Internal RAM.

- 32 Programmable I/O Lines.

- Eight Interrupt Sources.

- Full Duplex UART Serial Channel.

- Low-power Idle and Power-down Modes.

- Interrupt Recovery from Power-down Mode.

- Watchdog Timer.

- Dual Data Pointer.

- Power-off Flag.

- Fast Programming Time.

- $\quad$ Flexible ISP Programming (Byte and Page Mode).

\subsubsection{DESCRIPTION}

The AT89S52 is a low-power, high-performance CMOS 8-bit microcontroller with 8K bytes of insystem programmable Flash memory. The on-chip Flash allows the program memory to be reprogrammed in system or by a conventional nonvolatile memory programmer. By combining a versatile 8-bit CPU with insystem programmable Flash on a monolithic chip, the Atmel AT89S52 is a powerful microcontroller which provides a highly-flexible and cost-effective solution to many embedded control applications. The AT89S52 provides the following standard features: $8 \mathrm{~K}$ bytes of Flash, 256 bytes of RAM, 32 I/O lines, Watchdog timer, two data pointers, three 16-bit timer/counters, a six-vector two-level interrupt architecture, a full duplex serial port, on-chip oscillator, and clock circuitry. In addition, the AT89S52 is designed with static logic for operation down to zero frequency and supports two software selectable power saving modes. The Idle Mode stops the CPU while allowing the RAM, timer/counters, serial port, and interrupt system to continue functioning. 


\subsubsection{RADIO TRANSMITTER DESIGN}

It is a complex topic which can be broken down into a series of smaller topics. A radio communication system requires two tuned circuits each at the transmitter and receiver, all four tuned to the same frequency and the bandwidth of the system being maintained at uniform level. The transmitter is an electronic device which, usually with the aid of an antenna, propagates an electromagnetic signal such as radio, television, or other telecommunications.

\subsubsection{DESIGN METHODOLOGY} circuits:

At the beginning of the 20th century, there were four chief methods of arranging the transmitting

1. The transmitting system consists of two tuned circuits such that the one containing the spark-gap is a persistent oscillator; the other, containing the aerial structure, is a free radiator maintained in oscillation by being coupled to the first (Nikola Tesla and Guglielmo Marconi).

2. The oscillating system, including the aerial structure with its associated inductance-coils and condensers, is designed to be both a sufficiently persistent oscillator and a sufficiently active radiator (Oliver Joseph Lodge).

3. The transmitting system consists of two electrically coupled circuits, one of which, containing the air-gap, is a powerful but not persistent oscillator, being provided with a device for quenching the spark as soon as it has imparted sufficient energy to the other circuit containing the aerial structure, this second circuit then independently radiating the train of slightly damped waves at its own period (Oliver Joseph Lodge and Wilhelm Wien).

4. The transmitting system, by means either of an oscillating arc (Valdemar Poulsen) or a high frequency alternator (Rudolf Goldschmidt), emits a persistent train of undamped waves interrupted only by being broken up into long and short groups by the operator's key.

\subsubsection{FREQUENCY SYNTHESIS}

\subsubsection{FIXED FREQUENCY SYSTEMS}

For a fixed frequency transmitter one commonly used method is to use a resonant quartz crystal in a $\mathrm{RC}$ Crystal oscillator to fix the frequency on par with the resonant frequency. Where the frequency has to be variable, several options can be used.

\subsubsection{VARIABLE FREQUENCY SYSTEMS}

An array of quartz crystals is used to enable a transmitter to be used on several different frequencies rather than being a truly variable frequency system, it is a system which is fixed to several different frequencies (a subset of the above).

- Variable-frequency oscillator (VFO)

- $\quad$ PLL Frequency Synthesizer

\subsubsection{FREQUENCY MIXING AND MODULATION}

The task of many transmitters is to transmit some form of information using a radio frequency signal (carrier wave) which has been modulated to carry the intelligent required signal. A few rare types of transmitter do not carry information: the RF generator in a microwave oven, electro surgery, and induction heating. RF transmitters that do not carry information are being governed by the law to operate in Industrial Scientific and Medical (ISM) radio band.

\subsubsection{LINKING OF THE TRANSMITTER TO THE AERIAL}

The majority of modern transmitting equipment is designed to operate with a resistive load fed via a coaxial cable of particular characteristic impedance, often $50 \mathrm{ohms}$. To connect the aerial to this coaxial cable transmission line of normally a high impedance matching network and/or a balun may be required. Commonly an SWR meter and/or an antenna analyzer are used to check the extent of the match between the aerial system and the transmitter via the transmission line (feeder). An SWR meter indicates forward power, reflected power, and the ratio between them.

\subsubsection{WORKING OF AT89S52 MICROCONTROLLER}

Even though there are a large number of different types of microcontrollers and even more programs created for their use only, all of them have many things in common. Thus, if you learn to handle one of them you will be able to handle them all. A typical scenario on the basis of which it all functions is as follows:

- Power supply is turned off and everything is stand still. The program is loaded into the microcontroller, nothing indicates what is about to come. 
- Power supply is turned on and everything starts to happen at high speed. The control logic unit keeps everything under control.

- It disables all other circuits except quartz crystal to operate. While the preparations are in progress, the first milliseconds go by.

When power supply voltage attains its maximum value and the oscillator frequency becomes stable. SFRs are being filled with bits reflecting the state of all circuits within the microcontroller. All pins are configured as inputs. The overall electronics starts operation in rhythm with pulse sequence. From now on the time is measured in micro and nanoseconds. The program Counter is set to zero. Instruction from that address is sent to instruction decoder which recognizes it, after which it is executed with immediate effect. The value of the Program Counter is incremented by 1 and the whole process is repeated several million times per second.

\subsubsection{SERIAL COMMUNICATION}

One of the most important things concerning serial communication is the Protocol which should be strictly observed. It is a set of rules which must be applied in order that devices can correctly interpret data they mutually exchange. Fortunately, the microcontrollers automatically take care of this, so the work of the programmer/user is reduced to a simple write (data to be sent) and read (received data). A byte consists of 8 bits grouped together. If a bit is a digit then it is logical that bytes are numbers. All mathematical operations can be performed upon them, just like upon common decimal numbers, which is carried out in the ALU. It is important to remember that byte digits are not of equal significance. The largest value has the leftmost bit called the most significant bit (MSB). The rightmost bit has the least value and is therefore called the least significant bit (LSB). Since 8 digits (zeros and ones) of one byte can be combined in 256 different ways, the largest decimal number which can be represented by one byte is 255 (one combination represents zero).

\subsubsection{ULTRASONIC PULSE OSCILLATOR}

IC1 is the oscillation circuit to control the sending-out time of the ultrasonic pulse. The circuit is the same as the ultrasonic range meter but the value of the resistors and the capacitors are changed. The oscillation frequency is the same. The time of the oscillation pulse can be calculated by the following formula. Actually, with the occurrence of small error the calculation made is a bit different.

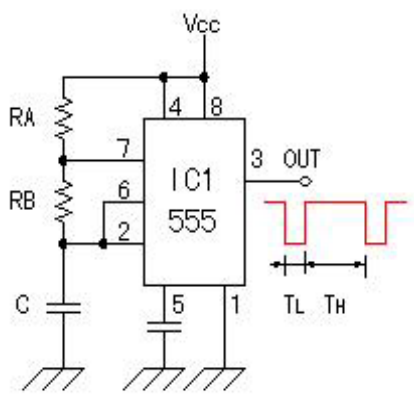

Fig 3.1.8.1 Circuit of Ultrasonic Sensor Pulse Oscillator

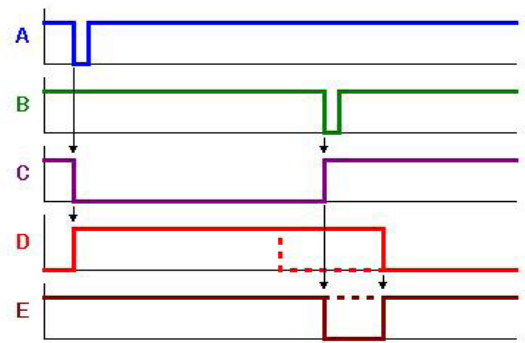

Fig 3.1.8.2 Waveform of Ultrasonic Sensor Pulse Oscillator

\section{DESIGN CALCULATIONS}

The condition: $\mathrm{RA}=1 \mathrm{M}-\mathrm{ohm}, \mathrm{RB}=15 \mathrm{~K}-\mathrm{ohm}, \mathrm{C}=0.1 \mu \mathrm{F}$

$\mathbf{T L}=0.69 \times \mathbf{R B} \times \mathbf{C}$

$=0.69 \times 15 \times 103 \times 0.1 \times 10-6$

$=1 \times 10-3$

$=1 \mathrm{msec}$

TH $=0.69 \times($ RA + RB $) \times \mathbf{C}$

$=0.69 \times 1015 \times 103 \times 0.1 \times 10-6$

$=70.0 \times 10-3$

$=70 \mathrm{msec}$

\subsubsection{LEVEL SENSOR}

Level sensors detect the level of substances that flow, including liquids, slurries, granular materials, and powders. All such substances flow to become essentially level in their containers (or other physical boundaries) because of gravity. The substance to be measured can be placed inside a container or can be in its natural form (e.g. a river or a lake).The level measurement can be either continuous or point values. Continuous 
level sensors measure level within a specified range and determine the exact amount of substance in a certain place, while point-level sensors only indicate whether the substance is above or below the sensing point. Generally the latter detect the levels that are excessively high or low. There are many physical and application variables that affect the selection of the optimal level monitoring method for industrial and commercial processes. The selection criteria include the physical: phase (liquid, solid or slurry), temperature, pressure or vacuum, chemistry, dielectric constant of medium, density (specific gravity) of medium, agitation (action), acoustical or electrical noise, vibration, mechanical shock, tank or bin size and shape. Also important are the application constraints: price, accuracy, appearance, response rate, ease of calibration or programming, physical size and mounting of the instrument, monitoring or control of continuous or discrete (point) levels.

\subsubsection{SELECTION OF LEVEL SENSOR}

- The MCX104A DC Microsyn Level Sensor electromagnetically measures the deviation of the sensor and its mount from a gravity reference.

- The reference is an oil damped pendulous mass having freedom of movement in one plane about a fixed center point.

- The sensor can be used as a component in a system requiring a reference for control with respect to gravity.

- It is ideal for use on mobile equipment where a surface must be finished to a required level with respect to gravity.

- In typical use, the sensor is connected to a controller, which controls a hydraulic control valve such as a KVF servo valve.

- If an off-level surface is required, an adjustable set point reference can be used, providing up to a $\pm 10 \%$ slope reference.

\subsubsection{FEATURES}

- Modular design: Compact unit can be mounted on any vertical surface, totally enclosed in a rugged cast aluminum housing.

- Easy to wire. Connections between controller and sensor are made through MS connectors.

- Excellent sensitivity. The shaft supporting the pendulous mass and the rotor of the Microsyn is mounted on ball bearings for smooth, low-friction rotation.

- Oil damped to ignore extraneous vibration.

- DC input and output voltages designed for analog and microcontroller interface.

Table 3.1.9 Technical datas related to the Level Sensor

\begin{tabular}{|l|l|}
\hline Input Voltage & 5 to 8 Vdc (Positive to Pin A, Negative to Pin C) \\
\hline Power Consumption & 0.8 Watts, Maximum \\
\hline Output Voltage & $25 \%$ of input to $75 \%$ of input from $-10 \%$ to $+10 \%$ Slope \\
\hline Range & $10 \%$ Slope from vertical, beyond $+/-10 \%$ range, output is undefined \\
\hline $\begin{array}{l}\text { Accuracy (Linearity) } \\
\text { Resolution }\end{array}$ & $\begin{array}{l}+/-0.3 \% \text { Slope from }+/-10 \% \text { Slope } \\
\text { Infinite }\end{array}$ \\
\hline Short Circuit Protection & Output lead may be short circuited to (+)or(-) input without damage to the device \\
\hline Repeatability (Hysteresis) & $0.04 \%$ slope, Maximum, when brought to the same position from (+) or (-) $10 \%$ slope \\
\hline Step Function Response & 0.42 s to reach $63 \%$ of rated output with 3000 Centistoke oil \\
\hline Phasing & $\begin{array}{l}\text { When the sensor is rotated clockwise from the null, as viewed from the close end the cast } \\
\text { housing, output (Pin B Positive, Pin C Negative) will decrease }\end{array}$ \\
\hline
\end{tabular}

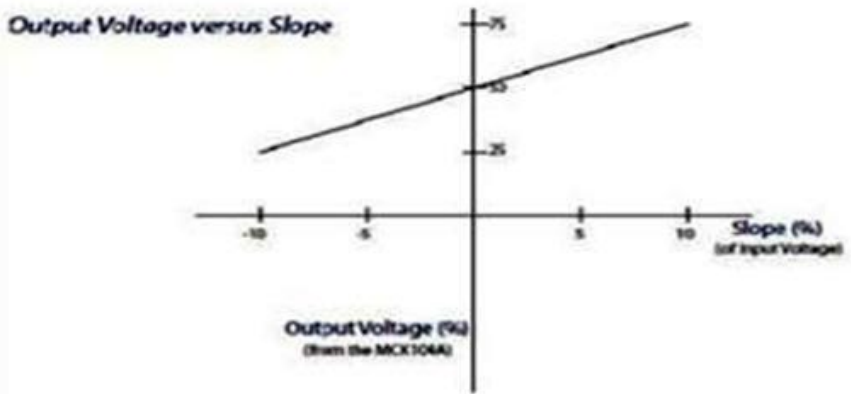

Fig 3.1.9 Waveform of the Level Sensor 


\subsubsection{SIMULATED RESULTS}
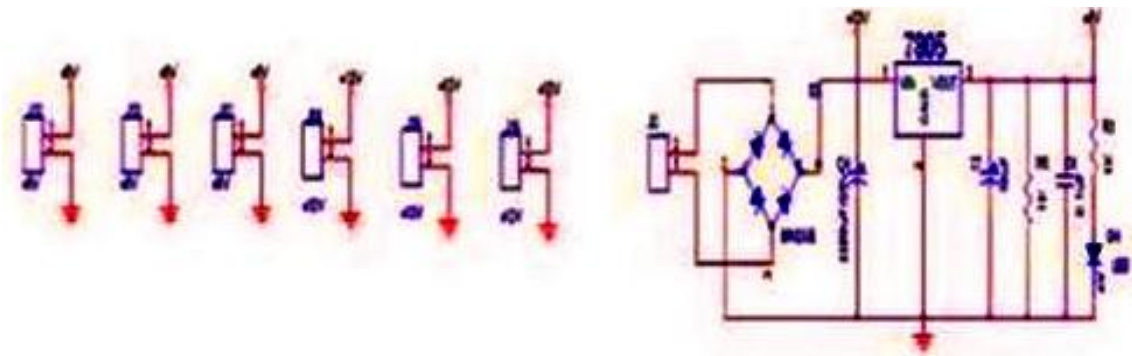

Fig 3.1.10.1 Circuit Diagram of Transmitter Section

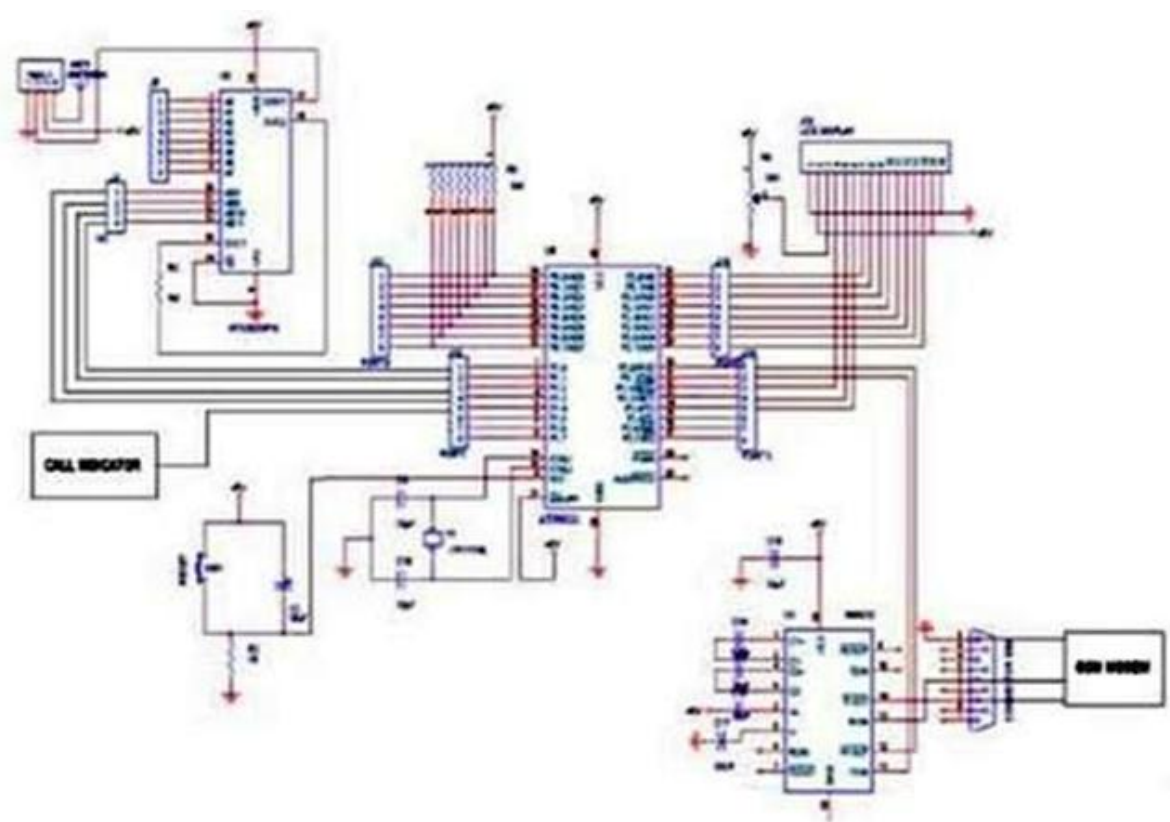

Fig 3.1.10.2 Circuit Diagram of Receiver Section

3.2 Experimental Results of the Proposed Hardware System

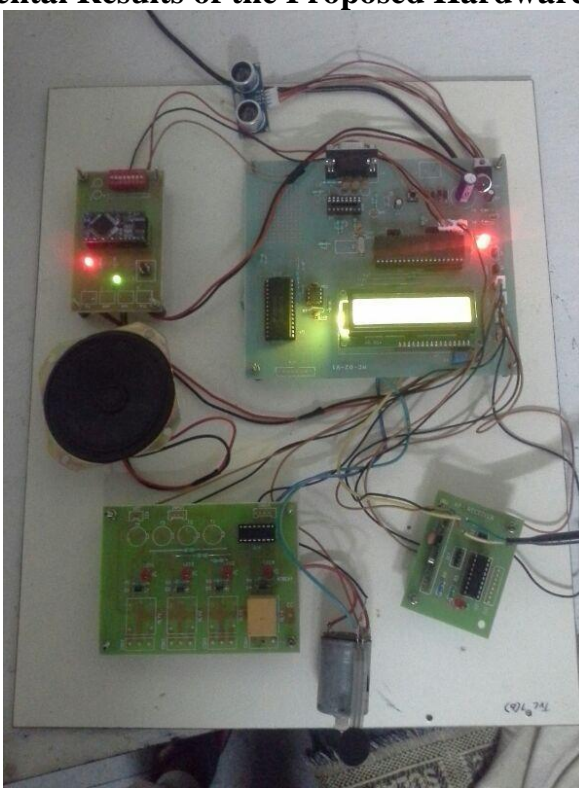

Fig 3.2.1 Hardware KIT TX Section

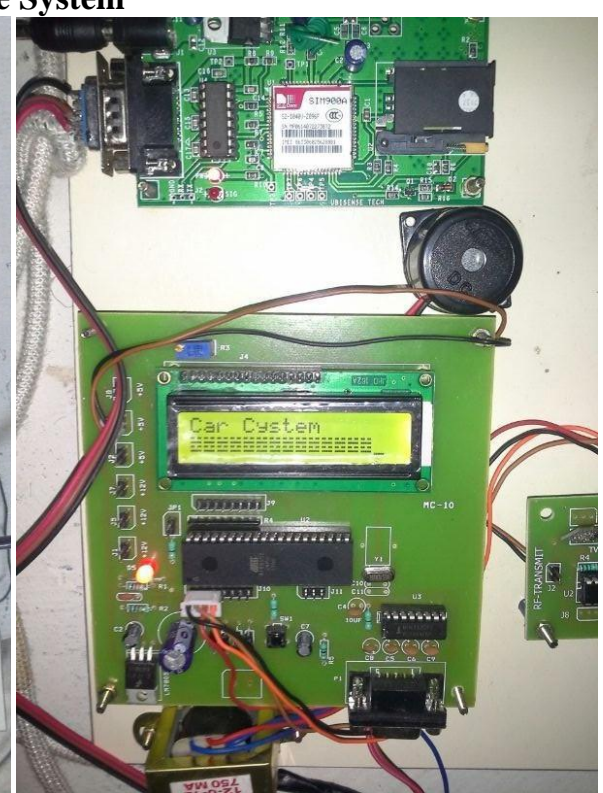

Fig 3.2.2 Hardware KIT RX Section 


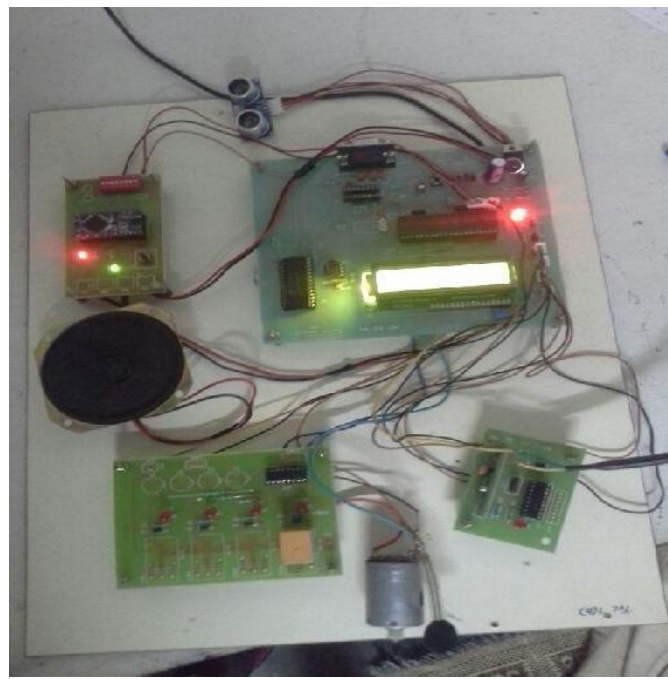

Fig 3.2.3 Grip Sensor Not Holded

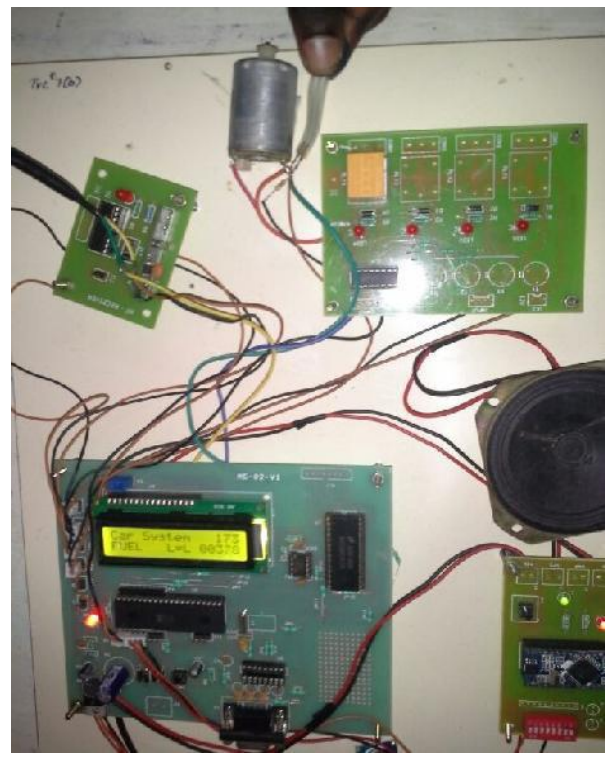

Fig 3.2.5 Fuel Sensor Level Low

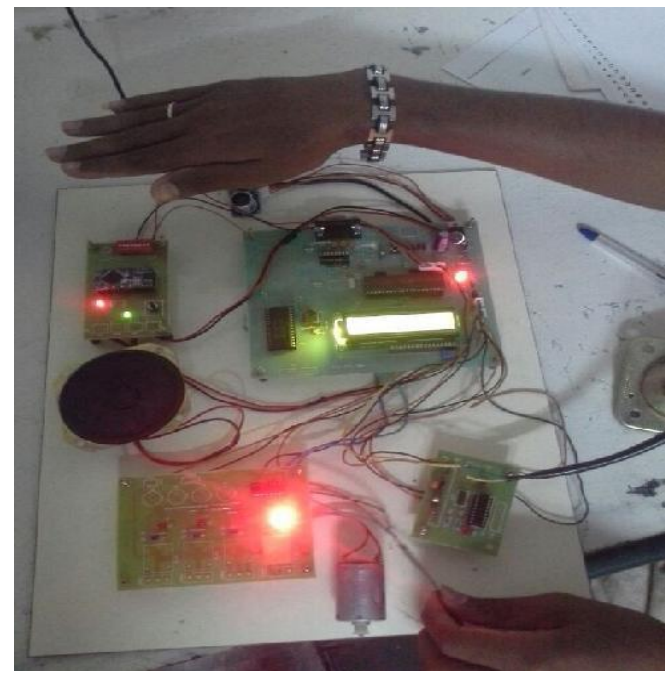

Fig 3.2.7 Object being sensed with Ultrasonic Sensor

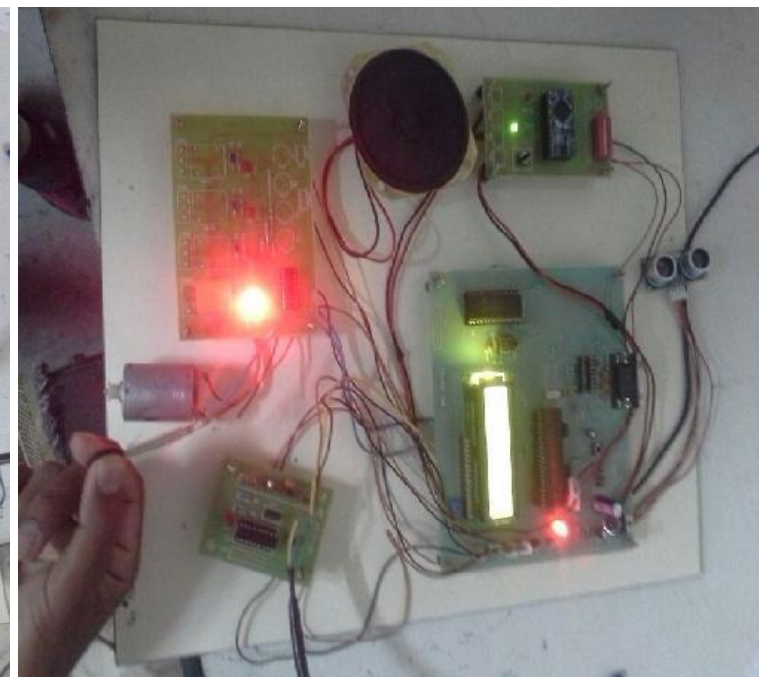

Fig 3.2.4 Grip Sensor Holded

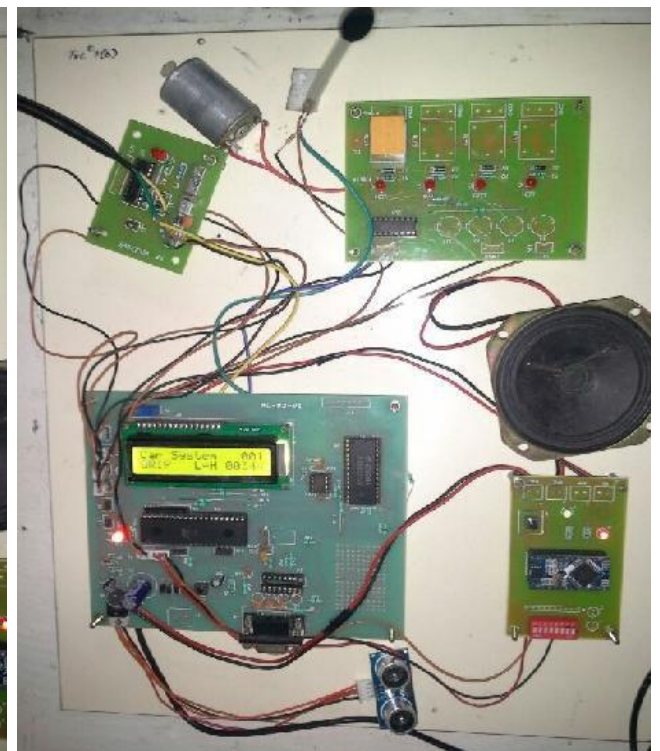

Fig 3.2.6 Fuel Sensor Level High

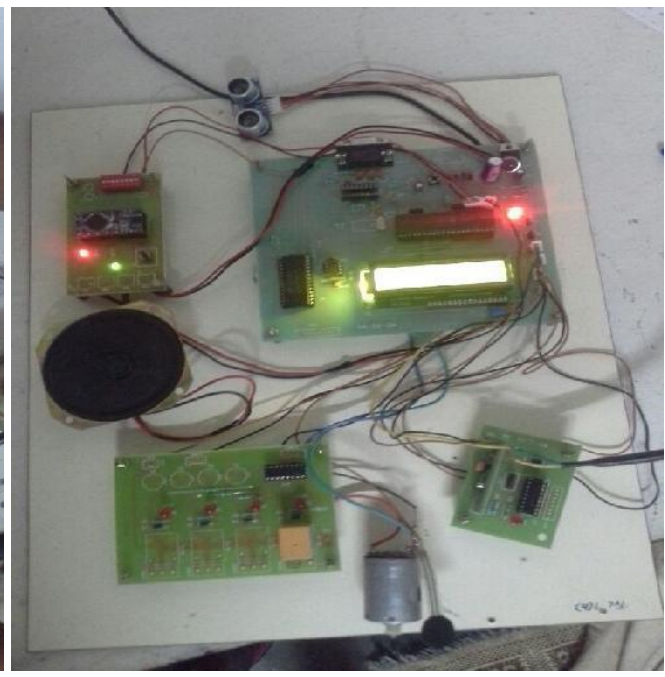

Fig 3.2.8 Object not being sensed with Ultrasonic Sensor 


\section{SOFTWARE ANALYSIS}

The main purpose of using the ATMEL microcontroller in our project is because it is a highperformance CMOS 8-bit microcontroller with $8 \mathrm{~K}$ bytes of in-system programmable Flash memory. By combining a versatile 8-bit CPU with in-system programmable Flash on a monolithic chip, the Atmel AT89S52 is a powerful microcontroller which provides a highly-flexible and cost-effective solution to many embedded control applications. The programs of the microcontroller have been written in Embedded C language and were compiled using KEIL, a compiler used for microcontroller programming. The communication between PC and the microcontroller was established using MAX 232 standard and those programs were also been executed in C language. The following programs are used at various stages for the mentioned functions. Serial communication in this program, the various special function registers of the microcontroller are set such that they can send and receive data from the $\mathrm{PC}$. This program uses the serial library to communicate with the ports.

\subsection{KEIL COMPILER}

The $\mathrm{C}$ programming language is a general-purpose, programming language that provides code efficiency, elements of structured programming, and a rich set of operators. $\mathrm{C}$ is not a big language and is not designed for any one particular area of application. Its generality combined with its absence of restrictions, makes $\mathrm{C}$ a convenient and effective programming solution for a wide variety of software tasks. Many applications can be solved more easily and efficiently with $\mathrm{C}$ rather than with other more specialized languages. The Cx51 Optimizing C Compiler is a complete implementation of the American National Standards Institute (ANSI) standard for the $\mathrm{C}$ language. $\mathbf{C x 5 1}$ is not a universal $\mathrm{C}$ compiler adopted for the 8051 target. It is a ground-up implementation dedicated to generate extremely fast and compact code for the 8051 microcontroller. C $x 51$ provides you the flexibility of programming in $\mathrm{C}$ and the code efficiency and speed of assembly language. Since $\mathbf{C x 5 1}$ is a cross compiler, some aspects of the $\mathrm{C}$ programming language and standard libraries are altered or enhanced to address the peculiarities of an embedded target processor.

\subsubsection{SUPPORT FOR ALL 8051 VARIANTS}

The 8051 family is one of the fastest growing Microcontroller Architectures. More than 400 device variants from various silicon vendors are readily available. New extended 8051 Devices, like the Philips 80C51MX architecture are dedicated for large application with several Mbytes code and data space. For optimum support of these different 8051 variants, Keil provides the several development tools that are listed below. A new output file format (OMF2) allows direct support of up to 16MB code and data space. The CX51 compiler is a variant of the C51 compiler that is design for the new Philips 80C51MX architecture.

\subsection{COMPILING WITH CX51}

This explains how to use $\mathbf{C x} \mathbf{5 1}$ to compile $\mathrm{C}$ source files and discusses the control directives you may specify. These directives allow you to perform several functions. For example:

- $\quad$ Direct $\mathbf{C x 5 1}$ to generate a listing file

- Control the information included in the object file

- $\quad$ Specify code optimization and memory models

\section{Running Cx51 from the Command Prompt}

To invoke the C51 or CX51 compiler, enter C51 or CX51 at the command prompt. On the command line, you must include the name of the $\mathrm{C}$ source file to be compiled, as well as any other necessary control directives required to compile the source file.

The format for the $\mathbf{C x} \mathbf{5 1}$ command line is shown below:

C51 source file_directives..._

CX51 source file_directives...

or:

C51@command file

CX51@command file

Where:

Source file is the name of the source program that needs to be compiled.

Directives are the one that is used to control the function of the compiler.

Command file is the name of a command input file that may contain source file and directives. A command file is used, when the Cx51 invocation line gets complex command and exceeds the limits of the Windows command prompt.

The following command line example invokes C51, specifies the source file SAMPLE.C, and uses the controls DEBUG, CODE, and PREPRINT. 


\section{C51 SAMPLE.C DEBUG CODE PREPRINT}

The $\mathbf{C} \mathbf{x} 51$ compiler displays the following information upon successful invocation and compilation.

C51 COMPILER V6.10 C51 COMPILATION COMPLETE. 0

WARNING(S), 0 ERROR(S)

\section{CONCLUSION}

The hardware and software design of an intelligent embedded control and monitoring system for real time collision avoidance applications is presented in this paper. The controlling mechanism of the various sensors was achieved using ATMEL AT89S52 Microcontroller. The Ultrasonic sensor, grip sensor and fuel level sensor and all the other sensor modules gives intimation to the driver through LCD and voice board. The $\mathrm{Ph}$ sensor sense the level of fuel and the RF Transmitter (TX)/Receiver (RX) Section is used for message sharing purpose. The GSM modem sends the message to the receiver section. The controlling process of the ATMEL AT89S52 was implemented in software using embedded C platform by using a KEIL complier. The entire mechanism is implemented in hardware. The Concept involved in the above design can be widely used for commercial purposes. This facile mechanism elucidates us how a Collision Avoidance system can be maneuvered and serve its purpose more efficiently in a car system.

\section{ACKNOWLEDGEMENTS}

We are extremely grateful to Mr. L.Subramanyam, Distinguished Scientist, Head Electrical Works Section (EWS) Indira Gandhi Center for Atomic Research, Kalpakkam for his consistent support and guidance throughout this work.

\section{REFERENCES}

[1]. M. Distner, M. Bengtsson, T. Broberg, and L. Jakobsson, "City safety — A system addressing rear-end collisions at low speeds," in Proc. 21st Enhanc. Safety Veh. Conf., Stuttgart, Germany, 2009, paper 09-0371.

[2]. J. Hillenbrand, A. Spieker, and K. Kroschel, "A multilevel collision mitigation approach-Its situation assessment, decision making, and performance tradeoffs,'IEEE Trans. Intell. Transp. Syst., vol. 7, no. 4, pp. 528-540, Dec. 2006.

[3]. A. Broadhurst, S. Baker, and T. Kanade, "A prediction and planning framework for road safety analysis, obstacle avoidance and driver information," in Proc. 11th World Congr. Intell. Transp. Syst., Oct. 2004, pp. 1-26.

[4]. J. Jansson and F. Gustafsson, "A framework and automotive application of collision avoidance decision making," Automatica, vol. 44, no. 9,pp. 2347-2351,Sep. 2008

[5]. M. Goodrich and E. Boer, "Designing human-centered automation: Tradeoffs in collision avoidance system design," IEEE Trans. Intell. Transp.Syst. vol. 1, no.1, pp. 40-54, Mar. 2000.

[6]. J.-E. Källhammar, K. Smith, J. Karlsson, and E. Hollnagel, “Shouldn't cars react as drivers expect?” in Proc. 4th Int. Driving Symp. Hum. Fact.Driver Assess., Traning Veh. Design, 2007, pp. 9-15.

[7]. A. Eidehall and L. Petersson, "Statistical threat assessment for general road scenes using Monte Carlo sampling," IEEE Trans. Intell. Transp. Syst., vol. 9, no.1, pp. 137-147, Mar. 2008

[8]. N. Kaempchen, B. Schiele, and K. Dietmayer, "Situation assessment of an autonomous emergency brake for arbitrary vehicle-tovehicle collision scenarios," IEEE Trans. Intell. Transp. Syst., vol. 10, no. 4, pp. 678-687, Dec. 2009.

[9]. M. Althoff, O. Stursberg, and M. Buss, "Model-based probabilistic collision detection in autonomous driving," IEEE Trans. Intell. Transp. Syst.,vol. 10, no. 2, pp. 299-310, Jun. 2009.

[10]. M. Brännström, E. Coelingh, and J. Sjöberg, "Model-based threat assessment for avoiding arbitrary vehicle collisions," IEEE Trans. Intell. Transp.Syst. vol. 11, no. 3, pp. 658-669, Sep. 2010.

[11]. J. McCall and M. Trivedi, "Driver behavior and situation aware brake assistance for intelligent vehicles," Proc. IEEE, vol. 95, no. 2, pp. 374-387, Feb.2007.

[12]. L. Hammarstrand, F. Sandblom, L. Svensson, and J. Sorstedt, "Extended object tracking using a radar resolution model," IEEE Trans. Aerosp. Electron. Syst., vol. 48, no. 3, pp. 2371-2386, Jul. 2012.

[13]. J. Sorstedt, L. Svensson, F. Sandblom, and L. Hammarstrand, "A new vehicle motion model for improved predictions and situation assessment,”IEEE Trans. Intell. Transp. Syst., vol. 12, no. 4, pp. 1209-1219, Dec. 2011.

[14]. Stephanie Lefevre, Ruzena Bajcsy, Christian Laugi, "Probabilistic Decision Making for Collision Avoidance Systems: Postponing Decisions" IEEE/RSJ International Conference on Intelligent Robots and Systems, 2013, Tokyo, Japan. 2013.

[15]. Annual statistical report, Eur. Road Safety Observatory, 2008.

[Online].Available: http://ec.europa.eu/transport/wcm/road_safety/erso/safetynet/content/safetynet.html

[16]. Data sheet of AT89S52 ATMEL microcontroller 1919D-MICRO-6/08 [online].Available: www.atmel.com/images/doc1919.pdf 